\title{
PROJECTIVE MODULES FOR FROBENIUS KERNELS AND FINITE CHEVALLEY GROUPS
}

\author{
ZONGZHU LIN AND DANIEL K. NAKANO
}

\section{INTRODUCTION}

1.1. Let $G$ be a connected reductive algebraic group scheme which is defined over the finite field $\mathbb{F}_{q}$ and $k$ be an algebraically closed field of characteristic $p$ (with $q=p^{r}$ ). Let $F^{r}: G \rightarrow G$ be the Frobenius morphism defined the $\mathbb{F}_{q}$-structure on $G$ and $G_{r}=\operatorname{Ker}\left(F^{r}\right)$ be the Frobenius kernel. Furthermore, let $G\left(\mathbb{F}_{q}\right)$ be the finite Chevalley group obtained by taking the fixed points of $G$ under $F^{r}$. For over forty years, there has been much work in finding relationships between the three module categories $\operatorname{Mod}(G), \operatorname{Mod}\left(G_{r}\right)$ and $\operatorname{Mod}\left(G\left(\mathbb{F}_{q}\right)\right)$. The reader is referred to [Hum] for a recent historical account.

If $M$ is a rational $G$-module one can consider the restriction of the action of $M$ to either $G_{r}$ (denoted by $\left.\left.M\right|_{G_{r}}\right)$ or $G\left(\mathbb{F}_{q}\right)\left(\left.M\right|_{G\left(\mathbb{F}_{q}\right)}\right)$. Parshall asked the following question in the Proceedings of the 1987 Arcata Conference on Finite Groups [P, 5.3]:

(1.1.1) If $M$ is a rational $G$-module such that $\left.M\right|_{G_{1}}$ is projective, is $\left.M\right|_{G\left(\mathbb{F}_{p}\right)}$ projective?

In 1999, the authors [LN] provided an affirmative answer to this question for all but a few cases when the field has very small characteristic. The question (1.1.1) became known as the "Parshall conjecture". The ideas used in verifying the conjecture involved formulating connections between the lower central $p$-series for the finite $p$-group $U\left(\mathbb{F}_{p}\right)$ where $U$ is the unipotent radical of a Borel subgroup $B$ and the associated graded algebra of the group algebra $k U\left(\mathbb{F}_{p}\right)$ after filtering by powers of the augmentation ideal. Once this relationship is made precise one can use the May spectral sequence along with results on nilpotent orbits and support varieties to prove the result.

The following question is a natural generalization to (1.1.1):

(1.1.2) If $M$ is a rational $G$-module such that $\left.M\right|_{G_{r}}$ is projective, is $\left.M\right|_{G\left(\mathbb{F}_{q}\right)}$ projective?

For the sake of consistency in this paper, an affirmative answer to question (1.1.2) will be called the "generalized Parshall conjecture". The aforementioned techniques used in [LN] do not readily extend to the case when $r \geq 2$ so an answer to (1.1.2) has been quite elusive in recent years. The main result of this paper is

Theorem. For any connected reductive algebraic group $G$ defined and split over the finite field $\mathbb{F}_{q}$, if $M$ is a rational $G$-module such that $\left.M\right|_{G_{r}}$ is projective, then $\left.M\right|_{G\left(\mathbb{F}_{q}\right)}$ projective.

Date: February 2006.

1991 Mathematics Subject Classification. Primary 17B55, 20G; Secondary 17B50.

Research of the first author was supported in part by NSF grant DMS-0200673.

Research of the second author was supported in part by NSF grant DMS-0400548. 
Note that our result settles the generalized Parshall conjecture for fields of any positive characteristic. Furthermore, our proof is of general interest because it entails investigating lifting projective $U_{r}$-modules to $U$.

We also consider the converse to the generalized Parshall conjecture. In general, the converse is false because one can take the Steinberg module and twist it $r$-times. This module remains projective over $G\left(\mathbb{F}_{q}\right)$, but is a direct sum of trivial modules over $G_{r}$, and is thus not projective over $G_{r}$. However, the converse does hold when we restrict to a certain bounded full subcategory of $G$-modules called $\mathcal{C}_{r}(G)$. This was shown to hold for $G_{1}$ in [LN, Thm. 4.4] by classifying the indecomposable modules in $\mathcal{C}_{1}(G)$ (for $G=\mathrm{SL}_{2}$ ) which was possible because $\mathcal{C}_{1}(G)$ has finite representation type. For $r \geq 2, \mathcal{C}_{r}(G)$ is of wild representation type so we need to employ other methods which were an outgrowth of work in $[\mathrm{CLN}]$. Applications of our results are presented at the end of this paper which unify many previously known results involving projectivity and periodicity of modules for Frobenius kernels and finite Chevalley groups.

1.2. Throughout this paper let $k$ be an algebraically closed field of characteristic $p>0$ which contains the finite field of $q$ elements $\mathbb{F}_{q}\left(q=p^{r}\right)$. Let $G$ be a connected reductive algebraic $k$-group defined and split over the field $\mathbb{F}_{q}$. For $r \geq 1$, let $G_{r}$ be the $r$ th Frobenius kernel of $G$ and $G\left(\mathbb{F}_{q}\right)$ be the finite Chevalley group.

Let $T$ be a maximal split torus and $\Phi$ be the root system associated to $(G, T)$. Moreover, let $\Phi^{+}$(resp. $\Phi^{-}$) be the set of positive (resp. negative) roots and $\Delta$ be the set of simple roots. Let $\alpha^{\vee}=2 \alpha /\langle\alpha, \alpha\rangle$ be the coroot corresponding to $\alpha \in \Phi$. For a generic simple root $\alpha, \omega_{\alpha}$ will denote the corresponding fundamental weight. Let $B$ be a Borel subgroup containing $T$ corresponding to the negative roots and $U$ be the unipotent radical of $B$. The Frobenius kernels for $B$ (resp. $U$ ) are $B_{r}$ (resp. $U_{r}$ ) and the corresponding finite groups are $B\left(\mathbb{F}_{q}\right)$ and $U\left(\mathbb{F}_{q}\right)$.

1.3. If $G$ is connected and reductive, then $G=Z^{0}(G)[G, G]$ with $[G, G]$ being semisimple, defined and split over $\mathbb{F}_{q}$. Since $G_{r}=Z^{0}(G)_{r}[G, G]_{r}$ with $Z^{0}(G)$ being a central torus, any $G_{r}$-module is projective over $G_{r}$ if and only if it is projective when restricted to $[G, G]_{r}$. On the other hand, we have $G\left(\mathbb{F}_{q}\right)=Z^{0}(G)\left(\mathbb{F}_{q}\right)[G, G]\left(\mathbb{F}_{q}\right)$. The group $Z^{0}(G)\left(\mathbb{F}_{q}\right)$ consists of elements whose orders are prime to $p$. Thus a $G\left(\mathbb{F}_{q}\right)$-module is projective if and only if it is projective over $[G, G]\left(\mathbb{F}_{q}\right)$. Therefore, in order to prove Theorem 1.1, it suffices to assume that $G$ is semisimple.

If $\pi: G \rightarrow \check{G}$ is a surjective algebraic group homomorphism of reductive algebraic groups defined and split over $\mathbb{F}_{q}$ with $\operatorname{Ker} \pi$ and $\operatorname{Ker}(d \pi)$ being both central, i.e., $\pi$ is central as in [B, 22.3]. Take a split Borel subgroup $B=T U$ of $G$ and $\check{B}=\check{T} \check{U}$ of $\check{G}$ respectively such that $\check{B}=\pi(B)$. Then $\pi: U \rightarrow \check{U}$ is an isomorphism [B, 22.4]. By [Jan, II, 9.4, 11.4 (1)] a $G$-module $M$ is projective for $G_{r}$ if and only if it is projective for $U_{r}$ and $U_{r}^{-}$. A similar statement holds for the group $\check{G}$. Hence, any $\check{G}$-module $M$ is projective for $\check{G}_{r}$ if and only if is projective for $G_{r}$.

Over the finite field $\mathbb{F}_{q}, \pi$ restricts to an isomorphism of a Sylow $p$-subgroup of $G\left(\mathbb{F}_{q}\right)$ to a Sylow $p$-subgroup of $\check{G}\left(\mathbb{F}_{q}\right)$. Hence, a $\check{G}\left(\mathbb{F}_{q}\right)$-module is projective if and only if it is projective for $G\left(\mathbb{F}_{q}\right)$. In combination with the above argument we can state the following proposition. 
Proposition. If Theorem 1.1 holds for any simply connected semisimple algebraic group $G$, then it holds for all connected reductive groups.

1.4. The arguments in the rest of paper applies directly to reductive groups. However, for notational simplicity, we will assume throughout the paper that $G$ is a connected and simply connected semisimple algebraic group. Let $\mathbb{E}$ be the Euclidean space associated with $\Phi$, and the inner product on $\mathbb{E}$ will be denoted by $\langle$,$\rangle . Moreover, let X(T)$ be the integral weight lattice obtained from the root system $\Phi$. The lattice $X(T)$ is the same as the character group of a split maximal torus $T$ under the simply-connectedness assumption. The set $X(T)$ has a partial ordering defined as follows: $\lambda \geq \mu$ if and only if $\lambda-\mu \in \sum_{\alpha \in \Delta} \mathbb{N} \alpha$. If $G$ is simple, let $\alpha_{0}$ to be the highest short root. Moreover, let $\rho \in X(T)$ be the half sum of positive roots and $w_{0}$ denote the long element of the Weyl group. The Coxeter number associated to an indecomposable root system $\Phi$ is $h=\left\langle\rho, \alpha_{0}^{\vee}\right\rangle+1$.

The dominant integral weights are defined as

$$
X(T)_{+}=\left\{\lambda \in X(T): 0 \leq\left\langle\lambda, \alpha^{\vee}\right\rangle \text { for all } \alpha \in \Delta\right\},
$$

and the set of $p^{r}$-restricted weights is

$$
X_{r}(T)=\left\{\lambda \in X(T): 0 \leq\left\langle\lambda, \alpha^{\vee}\right\rangle<p^{r} \text { for all } \alpha \in \Delta\right\} .
$$

The simple modules for $G$ are indexed by the set $X(T)_{+}$and denoted by $L(\lambda), \lambda \in X(T)_{+}$ with $L(\lambda)=\operatorname{soc}_{G} H^{0}(\lambda)$ where $H^{0}(\lambda)=\operatorname{Ind}_{B}^{G} \lambda$. A complete set of non-isomorphic simple $G_{r}$-modules and $G\left(\mathbb{F}_{q}\right)$ are easily obtained by taking $\left\{L(\lambda): \lambda \in X_{r}(T)\right\}$.

\section{Generalized Parshall Conjecture}

2.1. We begin by proving a general result describing the structure of modules whose restriction to a normal subgroup scheme is injective. Let $H$ be an algebraic $k$-group scheme and $N$ be a closed normal subgroup scheme of $H$ over $k$. Assume that every simple $N$-module $L$ extends to an $H$-module structure. For any $H$-module $M, \operatorname{Hom}_{N}(L, M) \cong\left(L^{*} \otimes M\right)^{N}$ is an $H$-submodule of $L^{*} \otimes M$ with $N$ acting on $\operatorname{Hom}_{N}(L, M)$ trivially (thus an $H / N$-module). Furthermore,

$$
\operatorname{Soc}_{N}(M) \cong \bigoplus_{L} L \otimes \operatorname{Hom}_{N}(L, M)
$$

as an $H$-submodule under the evaluation map $L \otimes \operatorname{Hom}_{N}(L, M) \rightarrow M$ defined by $v \otimes$ $f \mapsto f(v)$, which is an $H$-module homomorphism. Note that the sum is taken over the isomorphism classes of all simple $N$-modules.

Proposition. Let $N$ be a closed normal subgroup of an algebraic $k$-group scheme $H$. For each simple $N$-module $L$, let $Q(L)$ be the $N$-injective hull of $L$. We further assume that both $L$ and $Q(L)$ extends to $H$-module structures. If $P$ is any $H$-module such that $\left.P\right|_{N}$ is injective, then there is an isomorphism of $H$-modules

$$
P \cong \bigoplus_{L} Q(L) \otimes \operatorname{Hom}_{N}(L, P) .
$$

Proof. We first note that $\operatorname{Soc}_{N}\left(\oplus_{L}\left(Q(L) \otimes \operatorname{Hom}_{N}(L, P)\right)\right) \cong \oplus_{L}\left(L \otimes \operatorname{Hom}_{N}(L, P)\right)$ as $H$ module since $\operatorname{Hom}_{N}(L, P)$ is a trivial $N$-module. Since both $P$ and $\oplus_{L} Q(L) \otimes \operatorname{Hom}_{N}(L, P)$ 
are injective $N$-modules and have isomorphic $N$-socle, then they are isomorphic as $N$ modules. But here we want the isomorphism to be an $H$-module isomorphism.

Let us fix an irreducible $N$-module $L$. For each $f \in \operatorname{Hom}_{N}(L, P)$, let $\tilde{f}: Q(L) \rightarrow P$ be an $N$-module homomorphism extending $f$ in the following commutative diagram

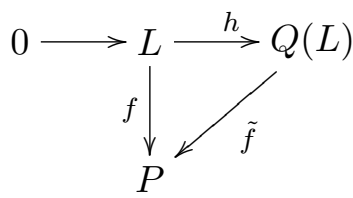

whose existence is guaranteed because $P$ is an injective $N$-module. Note that $h$ is an $H$ module homomorphism. The map $\tilde{f}$ is unique since $L$ is the $N$-socle of $Q(L)$ and the difference of any two $N$-module homomorphisms making the diagram commute would be zero when restricted to the socle $L=\operatorname{Soc}_{N}(Q(L))$ and thus has to be the zero map.

We now define $\phi_{L}: Q(L) \otimes \operatorname{Hom}_{N}(L, P) \rightarrow P$ by $\phi_{L}(v \otimes f)=\tilde{f}(v)$ which is well-defined and is clearly an $N$-module homomorphism.

For any $g \in H, g f \in \operatorname{Hom}_{N}(L, P)$ is defined by $(g f)(v)=g\left(f\left(g^{-1} v\right)\right)$ for all $v \in L$. Similarly, $g \tilde{f}$ is defined since $Q(L)$ is also an $H$-module. A direct verification using the fact that $h$ is an $H$-module homomorphism would yield $g f=\widetilde{g f} \circ h$ and $g f=g \tilde{f} \circ h$, i.e., the diagram

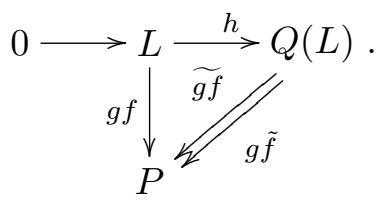

commutes using either one of the maps $\widetilde{g f}$ and $g \tilde{f}$. The uniqueness we mentioned above implies that $g \tilde{f}=\widetilde{g f}$. Thus we have

$$
\phi_{L}(g(v \otimes f))=\phi_{L}(g v \otimes g f)=\widetilde{g f}(g v)=(g \tilde{f})(g v)=g(\tilde{f}(v))=g\left(\phi_{L}(v \otimes f)\right)
$$

and $\phi_{L}$ is a homomorphism of $H$-modules. For two non-isomorphic simple $N$-modules $L$ and $L^{\prime}$, the submodules $\operatorname{Im}\left(\phi_{L}\right)$ and $\operatorname{Im}\left(\phi_{L^{\prime}}\right)$ have trivial intersection since their socles intersect trivially. Now the $H$-module homomorphism $\oplus_{L} \phi_{L}: \oplus_{L} Q(L) \otimes \operatorname{Hom}_{N}(L, P) \rightarrow P$ between the two injective $N$-modules must be an isomorphism because the restriction to the $N$-socles is an isomorphism.

2.2. The following result is standard and can be found in Jantzen's book [Jan, I. 3.10, I. 8.10].

Proposition. Let $A$ be a finite group scheme and $M$ be a finite dimensional $A$-module.

(a) $M$ is projective if and only if $M$ is injective;

(b) If $M$ is projective, then $M \otimes V$ is also projective for any A-module $V$.

2.3. Let $G$ be a connected reductive algebraic group scheme, $B$ a Borel subgroup of $G$ which is defined and split over $\mathbb{F}_{q}$ and $U$ the unipotent radical of $B$. In the next theorem we prove the generalized Parshall conjecture without any restrictions on the prime.

Theorem. Let $H=G, B$, or $U$ and $M$ be a rational $H$-module. If $M$ is projective as $H_{r}$-module, then $M$ is projective as $k H\left(\mathbb{F}_{q}\right)$-module. 
Proof. Let $M$ be a rational $H$-module. Proposition 2.2 implies that projectivity and injectivity are equivalent for the category of $H_{r}$-modules (resp. $H\left(\mathbb{F}_{q}\right)$-modules) because $H_{r}$ (resp. $H\left(\mathbb{F}_{q}\right)$ ) are finite group schemes.

We first take $H=U$. Clearly $U_{r}$ is normal in $U$. Consider the $r$ th Steinberg module $\mathrm{St}_{r}$ which is a $U$-module and is the $U_{r}$-injective hull of the only simple $U_{r}$-module $k$. We now apply Proposition 2.1 to our situation with $N=U_{r}$ and get a $U$-module isomorphism: $M \cong \mathrm{St}_{r} \otimes \operatorname{Hom}_{U_{r}}(k, M)$. Since $\mathrm{St}_{r}$ is injective as $U\left(\mathbb{F}_{q}\right)$-module, it follows that $M$ is injective (thus projective) over $k U\left(\mathbb{F}_{q}\right)$ (cf. Prop. 2.2).

For $H=B$, one uses [Jan, II. 9.4] to get that $M$ is projective as $B_{r}$-module if and only if it is projective as $U_{r}$-module and $M$ is projective as $B\left(\mathbb{F}_{q}\right)$-module if and only if it is projective as $k U\left(\mathbb{F}_{q}\right)$-module.

For $H=G$ one uses [Jan, II. 11.4] by considering a split Borel subgroup $B$ and its opposite $B^{+}$. Note that $U\left(\mathbb{F}_{q}\right)$ is a Sylow $p$-subgroup of $G\left(\mathbb{F}_{q}\right)$. Thus any $G\left(\mathbb{F}_{q}\right)$-module is projective if and only if it is projective over a Sylow $p$-subgroup. The above result for $U$ implies the result for $G$.

2.4. In the above argument, we used the properties that the Steinberg module $\mathrm{St}_{r} \cong$ $\operatorname{Ind}_{1}^{U_{r}} k \cong k\left[U_{r}\right]$ has a $U$-module structure and is projective as $U\left(\mathbb{F}_{q}\right)$-module. Observe that we did not actually use the fact that $\mathrm{St}_{r}$ has a $G$-module structure nor that it is projective as $G\left(\mathbb{F}_{q}\right)$-module. We conjecture the following result for arbitrary unipotent group schemes.

Conjecture. For any connected unipotent algebraic group scheme $U$ which is defined and split over $\mathbb{F}_{q}$, the $U_{r}$-module $\operatorname{Ind}_{1}^{U_{r}}(k)$ has a $U$-module structure such that $\operatorname{Ind}_{1}^{U_{r}}(k)$ is projective upon restriction to $U\left(\mathbb{F}_{q}\right)$.

The validity of the conjecture would imply that Theorem 2.3 would also holds for arbitrary connected split unipotent group $U$. Furthermore, one could use this result to extend Theorem 2.3 for arbitrary connected algebraic groups.

Corollary. Assuming the above conjecture is true, then for any connected algebraic group $G$ which is defined and split over $\mathbb{F}_{q}$ (i.e., there is a split Borel subgroup over $\mathbb{F}_{q}$ ), then any finite dimensional $G$-module $M$ which is projective over $G_{r}$ is also projective over $G\left(\mathbb{F}_{q}\right)$.

Proof. Take a split Borel subgroup $B$ with unipotent radical $U$. Then $U$ is defined and split over $\mathbb{F}_{q}$. The projectivity of $M$ over $G_{r}$ implies the projectivity over $U_{r}$. Thus $M$ is projective as a $U\left(\mathbb{F}_{q}\right)$-module. The unipotent radical of $G, \operatorname{Rad}_{u}(G)$, is a closed subgroup of $U$ and is defined and split over $\mathbb{F}_{q}$. Since $B / \operatorname{Rad}_{u}(G)$ is a split Borel subgroup of the reductive algebraic group $G / \operatorname{Rad}_{u}(G), U / \operatorname{Rad}_{u}(G)\left(\mathbb{F}_{q}\right)$ is a Sylow p-subgroup of $G / \operatorname{Rad}_{u}(G)\left(\mathbb{F}_{q}\right)=G\left(\mathbb{F}_{q}\right) / \operatorname{Rad}_{u}(G)\left(\mathbb{F}_{q}\right)$. This proves that $U\left(\mathbb{F}_{q}\right)$ is a Sylow $p$-subgroup of $G\left(\mathbb{F}_{q}\right)$. Hence, $M$ is a projective $G\left(\mathbb{F}_{q}\right)$-module.

2.5. When $p \geq 2(h-1)$, each indecomposable projective $G_{r}$-module $Q_{r}(\lambda)$ extends to a $G$-module. Jantzen showed this by constructing $Q_{r}(\lambda)$ as a $G$-direct summand of the tensor product of a Steinberg module $\mathrm{St}_{r}$ with another $G$-module. Such a realization implies that $Q_{r}(\lambda)$ is projective when restricted to the finite group $G\left(\mathbb{F}_{q}\right)$. However, it has been a long standing conjecture of Jantzen that $Q_{r}(\lambda)$ has a $G$-structure for all primes. The following corollary gives a different variation to this question. 
Corollary. Let $G$ be a connected reductive algebraic group defined and split over $\mathbb{F}_{q}$. Let $M$ be a finite dimensional projective $G_{r}$-module. If $M$ extends to a $G$-module structure, then $M$ is projective when restricted to the finite group $G\left(\mathbb{F}_{q}\right)$.

\section{Partial Converse to the Generalized Parshall Conjecture}

3.1. The Category $\mathcal{C}_{r}(G)$. In this section we still assume that $G$ is a connected, simplyconnected, semisimple algebraic group. The results in this section can easily be extended to reductive algebraic groups by using the arguments outlined in Section 1.3. Let $\mathcal{C}_{r}(G)$ be the full subcategory of rational $G$-modules whose composition factors have highest weights $\lambda$ satisfying $\left\langle\lambda, \alpha_{0}^{\vee}\right\rangle<p^{r}(p-1)$ for all highest short roots $\alpha_{0}$ of indecomposable components of the root system of $G$ with respect to a fixed split maximal torus. The category $\mathcal{C}_{r}(G)$ is a highest weight category and it is Morita equivalent to the module category for some quasi-hereditary algebra [CPS2].

3.2. We will make some comparisons between restricting certain elements in the distribution algebra $\operatorname{Dist}(G)$ of $G$. For this purpose, we recall the following result [CLN, Prop. $4.3]$.

Proposition . Let $M$ be a finite-dimensional vector space and $x, y \in \operatorname{End}_{k}(M)$ be two commuting elements such that $x \neq 0$ and $x^{p}=y^{p-1}=0$. If $M$ is a free module over the group algebra $k\langle 1+x\rangle$ then $M$ is a free module over the group algebra $k\langle 1+x+y\rangle$. Here $\langle 1+x\rangle$ and $\langle 1+x+y\rangle$ are cyclic groups of order $p$

3.3. We will now use the proposition above to analyze the case when $G=\mathrm{SL}_{2}$ with the intention of generalizing [CLN, Prop. 4.5]. In this case the unipotent radical $U$ is abelian. The distribution algebra $\operatorname{Dist}(U)$ has a basis $\left\{x^{(j)} \mid j=0,1,2, \ldots\right\}$, in which $\left\{x^{(j)} \mid j=0,1,2, \ldots, p^{r}-1\right\}$ is a basis of $\operatorname{Dist}\left(U_{r}\right)$ and $x^{(1)}$ is a basis element of the Lie algebra $\operatorname{Lie}(U)$. Let exp : $G_{a} \rightarrow U$ be the isomorphism of algebraic groups

$$
\exp (t)=\sum_{n=0}^{\infty} t^{n} x^{(n)}
$$

which provides the action on any rational $U$-module $M$ with $x^{(n)}$ acting as zero for sufficiently large $n$. The morphism exp is defined over the prime field $\mathbb{F}_{p}$.

One can express $\mathbb{F}_{q}=\mathbb{F}_{p}(\zeta)$ for some $\zeta$ with $\left\{1, \zeta, \zeta^{2}, \ldots, \zeta^{r-1}\right\}$ being a basis for $\mathbb{F}_{q}$ as an $\mathbb{F}_{p}$-vector space. Then $y_{i+1}=\exp \left(\zeta^{i}\right) \in U\left(\mathbb{F}_{q}\right)$. Then $\left\{y_{1}, \ldots, y_{r}\right\}$ forms a generating set for the elementary abelian group $U\left(\mathbb{F}_{q}\right)$ and

$$
U\left(\mathbb{F}_{q}\right)=\left\langle y_{1}\right\rangle \times\left\langle y_{2}\right\rangle \times \cdots \times\left\langle y_{r}\right\rangle .
$$

For $\alpha=\left(\alpha_{1}, \alpha_{2}, \ldots, \alpha_{r}\right) \in k^{r}$, set

$$
y_{\alpha}=\sum_{i=1}^{r} \alpha_{i}\left(y_{i}-1\right) \in k U\left(\mathbb{F}_{q}\right) .
$$

Any finite dimensional $U$-module $M$ restricts to a $k U\left(\mathbb{F}_{q}\right)$-module with $y_{\alpha}$ acting on $M$ via

$$
y_{\alpha}=\sum_{i=1}^{r} \alpha_{i}\left(\zeta^{i-1} x^{(1)}+\zeta^{2(i-1)} x^{(2)}+\ldots\right) .
$$


¿From the theory of rank varieties [Ben, Cor. 5.8.4], $M$ is projective over $U\left(\mathbb{F}_{q}\right)$ if and only if $\left.M\right|_{\left\langle 1+y_{\alpha}\right\rangle}$ is free for all $0 \neq \alpha \in k^{n}$. Note that $\left\langle 1+y_{\alpha}\right\rangle$ is a multiplicative cyclic subgroup of order $p$ in the group of invertible elements in $k U\left(\mathbb{F}_{q}\right)$.

On the other hand, for each $0 \neq \beta=\left(\beta_{1}, \beta_{2}, \ldots, \beta_{r}\right) \in k^{r}$ we define $z_{\beta}=\beta_{1} x^{(1)}+\beta_{2} x^{(p)}+$ $\cdots+\beta_{r} x^{\left(p^{r-1}\right)} \in \operatorname{Dist}\left(U_{r}\right)$. Then $z_{\beta}$ generates a 1-dimensional trivial restricted Lie algebra $k z_{\beta}$ with the restricted enveloping algebra $u\left(k z_{\beta}\right) \cong k\left\langle 1+z_{\beta}\right\rangle$, where $1+z_{\beta}$ generates a (multiplicative) cyclic subgroup $\left\langle 1+z_{\beta}\right\rangle$ of order $p$ in the algebra $u\left(k z_{\beta}\right)$. By [SFB], $M$ is projective over $U_{r}$ if and only if $\left.M\right|_{\left\langle z_{\beta}\right\rangle}$ is free (over the restricted enveloping algebra of the Lie algebra $\left\langle z_{\beta}\right\rangle$ for all $\beta \in k^{n}$ ). This is equivalent to $\left.M\right|_{\left\langle 1+z_{\beta}\right\rangle}$ being projective (cf. [Be, Cor. 3.1.4]).

Now assume that the following condition on $M$ holds:

$$
\left(x^{\left(p^{s}\right)}\right)^{p-1} M=0 \quad \text { for } s \geq r .
$$

We will show that $M$ is projective over $U_{r}$ if and only if $M$ is projective over $k U\left(\mathbb{F}_{q}\right)$.

By using Proposition 3.2 and condition (3.3.1), for any $\alpha=\left(\alpha_{1}, \cdots, \alpha_{r}\right) \in k^{r}$, the projectivity of $M$ over $\left\langle 1+y_{\alpha}\right\rangle$ is equivalent to the projectivity over $\left\langle 1+\tilde{y}_{\alpha}\right\rangle$ where

$$
\begin{aligned}
\tilde{y}_{\alpha}= & \sum_{i=1}^{r} \alpha_{i}\left(\zeta^{i-1} x^{(1)}+\zeta^{p(i-1)} x^{(p)}+\ldots \zeta^{p^{r-1}(i-1)} x^{\left(p^{r-1}\right)}\right) \\
= & \left(\alpha_{1}+\alpha_{2} \zeta+\cdots+\alpha_{r} \zeta^{r-1}\right) x^{(1)}+\left(\alpha_{1}+\alpha_{2} \zeta^{p}+\cdots+\alpha_{r} \zeta^{(r-1) p}\right) x^{(p)} \\
& +\cdots+\left(\alpha_{1}+\alpha_{2} \zeta^{p^{r-1}}+\cdots+\alpha_{r} \zeta^{(r-1) p^{r-1}}\right) x^{\left(p^{r-1}\right)} .
\end{aligned}
$$

Consider the matrix $A$ defined as

$$
A=\left(\begin{array}{cccc}
1 & \zeta & \ldots & \zeta^{r-1} \\
1 & \zeta^{p} & \ldots & \zeta^{(r-1) p} \\
\vdots & \vdots & \ddots & \vdots \\
1 & \zeta^{p^{r-1}} & \ldots & \zeta^{(r-1) p^{r-1}}
\end{array}\right)
$$

Since $\zeta, \zeta^{p}, \ldots, \zeta^{p^{r-1}}$ are $r$ distinct points in $k$ and $A$ is a Vandermonde matrix, it follows that $A$ is invertible. Hence $A$ defines a linear isomorphism $A: k^{r} \rightarrow k^{r}$ of vector spaces and $\tilde{y}_{\alpha}=z_{A(\alpha)}$. This shows that if condition (3.3.1) holds then $M$ is projective over $k U\left(\mathbb{F}_{q}\right)$ if and only if $M$ is projective over $U_{r}$.

Theorem. Let $G=\mathrm{SL}_{2}$ and $M \in \mathcal{C}_{r}\left(\mathrm{SL}_{2}\right)$. Then $M$ is projective over $\left(\mathrm{SL}_{2}\right)_{r}$ if and only if $M$ is projective over $k \mathrm{SL}_{2}\left(\mathbb{F}_{q}\right)$.

Proof. If $M$ is projective over $\left(\mathrm{SL}_{2}\right)_{r}$ then $M$ is projective over $k \mathrm{SL}_{2}\left(\mathbb{F}_{q}\right)$ by Proposition 2.3. On the other hand, if $M$ is in $\mathcal{C}_{r}\left(\mathrm{SL}_{2}\right)$ then the weights of $M$ are such that $\left\langle\lambda, \alpha^{\vee}\right\rangle\left\langle p^{r}(p-1)\right.$. One can now use a weight argument to show that (3.3.1) holds, i.e., $\left(x_{\alpha}^{\left(p^{s}\right)}\right)^{p-1} \cdot M=0$ for $s \geq r$. If $M$ is projective over $k \mathrm{SL}_{2}\left(\mathbb{F}_{q}\right)$ then $M$ is projective over $k U\left(\mathbb{F}_{q}\right)$, thus projective over $U_{r}$ from the argument above. It follows that $M$ is projective over $G_{r}$ by [CPS1, Main. Thm.]. 
3.4. We now return to a general semisimple group $G$. For modules in $\mathcal{C}_{r}(G)$, we can now show that the converse to the Parshall conjecture is valid.

Theorem. Let $M$ be a module in $\mathcal{C}_{r}(G)$. If $M$ is projective over $k G\left(\mathbb{F}_{q}\right)$ then $M$ is projective over $G_{r}$.

Proof. Let $M$ be in $\mathcal{C}_{r}(G)$ and $\left.M\right|_{G\left(\mathbb{F}_{q}\right)}$ be projective. Then $\left.M\right|_{U_{\alpha}\left(\mathbb{F}_{q}\right)}$ is projective for all $\alpha \in \Phi$. For $\alpha \in \Phi$, let $L_{\alpha}$ be the subgroup in $G$ generated by $U_{\alpha}$ and $U_{-\alpha}$. The subgroup $L_{\alpha}$ is defined and split over $\mathbb{F}_{q}$ and is reductive of type $A_{1}$. Since $M \in \mathcal{C}_{r}(G)$, it follows that $M$ is in $\mathcal{C}_{r}\left(L_{\alpha}\right)$ because $\left\langle\lambda, \alpha^{\vee}\right\rangle \leq\left\langle\lambda, \alpha_{0}^{\vee}\right\rangle\left\langle p^{r}(p-1)\right.$ for any dominant weight $\lambda$, any positive root $\alpha$ and the highest short root $\alpha_{0}$ of the component containing $\alpha$. By Theorem 3.3 , it follows that $\left.M\right|_{\left(U_{\alpha}\right)_{r}}$ is projective for all $\alpha \in \Phi$. Consequently, $\left.M\right|_{G_{r}}$ is projective by [CPS1, Main Thm.]

\section{Applications}

4.1. Cline, Parshall and Scott [CPS1, Main Thm.] proved that a finite-dimensional $G_{r} T$ module is projective if and only if it is projective over $\left(U_{\alpha}\right)_{r}$ for all $\alpha \in \Phi$. Friedlander and Parshall demonstrated later using support varieties that a rational finite-dimensional $G$ module is projective over $G_{1}$ if and only if it is projective over $\left(U_{\alpha}\right)_{1}$ where $\alpha$ is a long root. As an application of our techniques we provide a criterion for projectivity of a $G$-module over $k G\left(\mathbb{F}_{q}\right)$ via root subgroups. Let $\Gamma$ be a set of complete $W$-orbit representatives in $\Phi$. If $G$ is simple, then $\Gamma$ can be any set of one or two roots of different root lengths depending on whether the root system is simply laced or not.

Theorem. Let $G$ be a connected reductive algebraic group defined and split over $\mathbb{F}_{q}$. For any $M$ in $\mathcal{C}_{r}(G)$, the following conditions are equivalent.

(a) $\left.M\right|_{G_{r}}$ is projective;

(b) $\left.M\right|_{G\left(\mathbb{F}_{q}\right)}$ is projective;

(c) $\left.M\right|_{U_{\alpha}\left(\mathbb{F}_{q}\right)}$ is projective for all $\alpha \in \Phi$;

(d) $\left.M\right|_{U_{\alpha}\left(\mathbb{F}_{q}\right)}$ is projective for all $\alpha \in \Gamma$.

Proof. The implication $(a) \Rightarrow(b)$ follows from Theorem 2.3 , while $(b) \Rightarrow(a)$ holds because of Theorem 3.4. Now $U_{\alpha}\left(\mathbb{F}_{q}\right)$ is a subgroup of $G\left(\mathbb{F}_{q}\right)$ so every projective module over $k G\left(\mathbb{F}_{q}\right)$ is projective over $k U\left(\mathbb{F}_{q}\right)$. Therefore, we have $(b) \Rightarrow(c) \Rightarrow(d)$. It remains to show that $(d) \Rightarrow(a)$.

Assume that $\left.M\right|_{U_{\alpha}\left(\mathbb{F}_{q}\right)}$ is projective for all $\alpha \in \Gamma$. Since $M$ is a $G$-module it follows that $\left.M\right|_{U_{\alpha}\left(\mathbb{F}_{q}\right)}$ is projective for all $\alpha \in W(\Gamma)$ where $W(\Gamma)$ is the image of $\Gamma$ under the (ordinary) action of $W$. But, every root is $W$ conjugate to either a long or a short root so $W(\Gamma)=\Phi$. Now the fact that $M \in \mathcal{C}_{r}(G)$ implies that $\left.M\right|_{\left(U_{\alpha}\right)_{r}}$ is projective for all $\alpha \in \Phi$, thus by [CPS1, Main Thm.], $M$ is projective over $G_{r}$.

4.2. The theorem in the preceding section can be used to recover and generalize a theorem of Alperin and Mason [AM2].

Corollary (A). Let $L$ be a simple $G\left(\mathbb{F}_{q}\right)$-module with $p \geq h$. Suppose that $\left.L\right|_{U_{\alpha}\left(\mathbb{F}_{q}\right)}$ is projective for all $\alpha \in \Gamma$ (as defined in 4.1). Then $L \cong \mathrm{St}_{r}$. 
Proof. The $p \geq h$ condition insures that all simple modules $L$ are contained in $\mathcal{C}_{r}(G)$ since for any $\lambda \in X_{r}$, one has

$$
\left\langle\lambda, \alpha_{0}^{\vee}\right\rangle \leq\left\langle\left(p^{r}-1\right) \rho, \alpha_{0}^{\vee}\right\rangle \leq\left(p^{r}-1\right)(h-1)<p^{r}(p-1) .
$$

Let $L$ be a simple $G\left(\mathbb{F}_{q}\right)$-module. The hypotheses on $L$ and Theorem 4.1 implies that $L$ is a projective $G\left(\mathbb{F}_{q}\right)$-module and projective $G_{r}$-module. Consequently, $L \cong \mathrm{St}_{r}$ because $\mathrm{St}_{r}$ is the only simple projective $G_{r}$-module.

Theorem 4.1 can also be used to prove a result involving tensor products of modules for finite Chevalley groups which was proved earlier for $G_{1}$ by Friedlander and Parshall [FP, (2.4) Prop.].

Corollary (B). Let $M$ and $V$ be rational $G$-modules such that $M \otimes V$ is in $\mathcal{C}_{r}(G)$. If $\left.M \otimes V\right|_{G\left(\mathbb{F}_{q}\right)}$ is projective then either $\left.M\right|_{G\left(\mathbb{F}_{q}\right)}$ or $\left.V\right|_{G\left(\mathbb{F}_{q}\right)}$ is projective.

Proof. Suppose that $M \otimes V$ in $\mathcal{C}_{r}(G)$ and $\left.M \otimes V\right|_{G\left(\mathbb{F}_{q}\right)}$ is projective. According to Theorem 4.1, $\left.(M \otimes V)\right|_{G_{r}}$ is projective, and by [SFB], $\left.M\right|_{G_{r}}$ or $\left.V\right|_{G_{r}}$ is projective. Consequently, one sees that $\left.M\right|_{G\left(\mathbb{F}_{q}\right)}$ or $\left.V\right|_{G\left(\mathbb{F}_{q}\right)}$ is projective by Theorem 2.3.

4.3. For any finite-dimensional $k G\left(\mathbb{F}_{q}\right)$-module, one can associate an affine homogeneous variety, $V_{G\left(\mathbb{F}_{q}\right)}(M)$, often referred to as the support variety of $M$. A module $M$ over $k G\left(\mathbb{F}_{q}\right)$ is periodic if and only if it admits a periodic projective resolution. Every projective module is periodic, but a periodic module need not be projective, e.g., if $\operatorname{dim} V_{G\left(\mathbb{F}_{q}\right)}(M)=1$. Even though, our results so far have only been for projective modules over $G_{r}$ and $G\left(\mathbb{F}_{q}\right)$, we shall demonstrate that our techniques are powerful enough to make conclusions about the existence of periodic modules.

Theorem. Let $G$ be a simple algebraic group. If there exists $M$ in $\mathcal{C}_{r}(G)$ such that $M$ is indecomposable, periodic, non-projective $G\left(\mathbb{F}_{q}\right)$-module then $\operatorname{rank} G=1$.

Proof. First observe that if $M$ is periodic over $G\left(\mathbb{F}_{q}\right)$ and non-projective, it follows that $M_{U_{\alpha}\left(\mathbb{F}_{q}\right)}$ is not projective for some $\alpha \in \Phi$ because of Theorem 4.1. Since $M$ is periodic over $G\left(\mathbb{F}_{q}\right)$ this forces $M$ to be periodic over $U_{\alpha}\left(\mathbb{F}_{q}\right)$.

Now $M$ is a $G$-module and the Weyl group $W$ permutes the root subgroups so $M$ is periodic over $U_{w(\alpha)}\left(\mathbb{F}_{q}\right)$ for all $w \in W$. The indecomposability of $M$ over $G\left(\mathbb{F}_{q}\right)$ implies that $\operatorname{Proj}\left(V_{G\left(\mathbb{F}_{q}\right)}(M)\right)$ is connected. Suppose that $G$ has rank at least two. For each root length, there exist two different positive roots $\alpha$ and $\beta$ of the same length such that $U_{\alpha}\left(\mathbb{F}_{q}\right)$ and $U_{\beta}\left(\mathbb{F}_{q}\right)$ generate, in $G\left(\mathbb{F}_{q}\right)$, an elementary abelian subgroup $U_{\alpha, \beta}\left(\mathbb{F}_{q}\right) \cong U_{\alpha}\left(\mathbb{F}_{q}\right) \times U_{\beta}\left(\mathbb{F}_{q}\right)$. This can see by inspecting the roots systems by looking for two positive roots $\alpha, \beta$ of the same length such that $\alpha+\beta$ is not a root (taking one of them to be highest long/short root). In particular, choose the $\alpha$ and $\beta$ such that $M$ is not projective over $U_{\alpha}\left(\mathbb{F}_{q}\right)$ and $U_{\beta}\left(\mathbb{F}_{q}\right)$. Thus $V_{U_{\alpha}\left(\mathbb{F}_{q}\right) \times U_{\beta}\left(\mathbb{F}_{q}\right)}(M)$ contains two different affine lines. This shows that $\operatorname{res}^{*}\left(V_{U_{\alpha, \beta}\left(\mathbb{F}_{q}\right)}(M)\right) \subset$ $V_{G\left(\mathbb{F}_{q}\right)}(M)$ contains two different affine lines $\mathbb{A}^{1}$. But, the connectivity and the fact that $\operatorname{dim} V_{G\left(\mathbb{F}_{q}\right)}(M)$ is one-dimensional shows that $V_{G\left(\mathbb{F}_{q}\right)}(M)$ is an affine line. This contradiction implies that rank $G=1$.

As an corollary one can immediately deduce the main result of [JJ] for $p \geq h$ (cf. proof of Cor. 4.2(A)). 
Corollary. Let $G$ be a simple algebraic group with $p \geq h$. Suppose that $L$ is a simple periodic non-projective $G\left(\mathbb{F}_{q}\right)$-module. Then $\operatorname{rank} G=1$.

\section{REFERENCES}

[AM1] J. L. Alperin, G. Mason, On simple modules for $S L(2, q)$, Bull. London Math. Soc. 25 (1993), no. $1,17-22$.

[AM2] J. L. Alperin, G. Mason, Partial Steinberg modules for finite groups of Lie type, Bull. London Math. Soc. 25 (1993), no. 6, 553-557.

[Be] C. P. Bendel, Support varieties for infinitesimal algebraic groups, Ph.D thesis, Northwestern University, 1996.

[Ben] D. J. Benson, Representations and cohomology. II. Cohomology of groups and modules. Cambridge Studies in Advanced Mathematics, 31. Cambridge University Press, Cambridge, 1991.

[B] A. Borel, Linear Algebraic Groups Second Enlarged Edition, Springer-Verlag, New York, 1991

[CLN] J. F. Carlson, Z. Lin, D. K. Nakano, Support varieties for modules over Chevalley groups and classical Lie algebras, to appear in Trans. Amer. Math. Soc.

[CLNP] J. F. Carlson, Z. Lin, D. K. Nakano, B. J. Parshall, The restricted nullcone, Cont. Math. 325 (2003), 51-75.

[C] R. W. Carter, Finite groups of Lie type, Wiley-Interscience, 1985.

[CPS1] E. Cline, B. Parshall, L. Scott, On injective modules for infinitesimal algebraic groups I, J. London Math. Soc. (2) 31 (1985), 277-291.

[CPS2] E. Cline, B. Parshall, L. Scott, Finite-dimensional algebras and highest weight categories. J. Reine Angew. Math. 391 (1988), 85-99.

[CPSvdK] E. Cline, B. Parshall, L. Scott, W. van der Kallen, Rational and generic cohomology Invent. Math. 39 (1977), 143-163.

[FP] E. M. Friedlander, B. J. Parshall, Support varieties for restricted Lie algebras, Invent. Math. 86 (1986), 553-562.

[FPe] E. M. Friedlander, J. Pevtsova, Representation theoretic support spaces for finite group schemes, Amer. J. Math. 127 (2005), 379-420.

[FS] E. M. Friedlander, A. A. Suslin, Cohomology of finite group schemes over a field, Invent. Math. 127 (1997), no. 2, 209-270.

[Hum] J. E. Humphreys, Modular representations of finite groups of Lie type, London Math. Soc. Lecture Note Series 326, Cambridge University Press, 2005.

[JJ] I. Janiszczak, J. C. Jantzen, Simple periodic modules over Chevalley groups, J. London Math. Soc. 41 (1990), 217-230.

[Jan] J. C. Jantzen, Representations of Algebraic Groups, 2nd Edition, Math. Survey and Mono., Vol. 107, American Math. Soc., 2003.

[LN] Z. Lin, D. K. Nakano, Complexity for modules over finite Chevalley groups and classical Lie algebras, Invent. Math. 138 (1999), 85-101.

[NPV] D. K. Nakano, B. J. Parshall, D. C. Vella, Support varieties for algebraic groups, J. Reine Angew. Math. 547 (2002), 15-47.

[P] B. J. Parshall, Cohomology of algebraic groups, Proc. Symp. Pure Math., 47 (1987), 233-248.

[SFB] A. Suslin, E. Friedlander, C. Bendel, Support varieties for infinitesimal group schemes, J. Amer. Math. Soc. 10, (1997), 729-759.

Department of Mathematics, Kansas State University, Manhattan, KS 66506

E-mail address: zlin@math.ksu.edu

Department of Mathematics, University of Georgia, Athens, GA 30602

E-mail address: nakano@math.uga.edu 